\title{
"Doing it Right" Overnight: a Multi-perspective Qualitative Study Exploring Senior Medical Resident Overnight Call
}

\author{
Sarah Burm, $P h D^{7} \oplus$, Saad Chahine, $P h D^{2}$, and Mark Goldszmidt, MD, PhD, FRCPC ${ }^{3}$ \\ ${ }^{1}$ Continuing Professional Development/Division of Medical Education, Faculty of Medicine, Dalhousie University, Room 2L-23 Sir Charles Tupper \\ Medical Building, 5850 College Street, Halifax, Nova Scotia, Canada; ${ }^{2}$ Faculty of Education, Queen's University, Kingston, Ontario, Canada; \\ ${ }^{3}$ Division of General Internal Medicine, Department of Medicine, Centre for Education Research and Innovation, Western University, London, \\ Ontario, Canada.
}

BACKGROUND: Competency-based medical education (CBME) requires the development of workplace-based assessment tools that are grounded in authentic clinical work. Developing such tools, however, requires a deep understanding of the underlying facets of the competencies being assessed. Gaining this understanding remains challenging in contexts where performance is not readily visible to supervisors such as the senior medical resident (SMR) on-call role in internal medicine.

OBJECTIVE: This study draws on the perspectives of healthcare professionals with whom the SMR interacts with overnight to generate insights into the different components of on-call SMR practice and the range of ways SMRs effectively and less effectively enact these.

APPROACH: We used a constructivist grounded theory (CGT) approach to examine variation in how on-call SMRs carry out their role overnight.

PARTICIPANTS: Six medical students, five junior residents, five internal medicine attending physicians, five emergency physicians, and three emergency nurses conducted observations of their on-call interactions with SMRs. Participants were then interviewed and asked to elaborate on their observations as well as provide comparative reflections on the practices of past SMRs they worked with.

KEY RESULTS: Strong collaboration and organizational skills were identified as critical components to effectively being the on-call SMR. Perceived weaker SMRs, while potentially also having issues with clinical skills, stood out more when they could not effectively manage the realities of collaboration in a busy workplace.

CONCLUSION: What consistently differentiated a perceived effective SMR from a less effective SMR was someone who was equipped to manage the realities of interprofessional collaboration in a busy workplace. Our study invites medical educators to consider what residents, particularly those in more complex roles, need to receive

This research was presented at The American Education Research Association's (AERA) annual conference in April 2018 and The Association for Medical Education in Europe (AMEE) annual conference in August 2017.

Electronic supplementary material The online version of this article (https://doi.org/10.1007/s11606-020-06284-1) contains supplementary material, which is available to authorized users.

Received February 6, 2020

Accepted October 5, 2020

Published online October 19, 2020 feedback on to support their development as physicians. It is our intention that the findings be used to inform the ways programs approach teaching, assessment, and the provision of feedback.

KEY WORDS: competency; on-call supervision; medical education; qualitative research.

J Gen Intern Med 36(4):881-7

DOI: $10.1007 / \mathrm{s} 11606-020-06284-1$

(c) Society of General Internal Medicine 2020

\section{INTRODUCTION}

Essential to the success of competency-based medical education (CBME) will be the design of robust workplace-based assessment grounded in authentic clinical work. ${ }^{1,2}$ However, as roles take on increasing complexity, it can be difficult to fully understand the components of practice we need to cultivate, assess, and provide feedback to residents on. This is especially true when discussing transition roles - roles where you move from one level of responsibility to another. Specifically, in internal medicine (IM), July 1 marks the beginning of a new academic year, where residents transition from the junior role post-graduate year one (PGY 1) to the senior role (PGY 2). With this shift come expectations of functioning as a senior resident and assuming greater responsibility. This aspect of IM training means in many centers, overnight call changes; instead of doing task execution under direct observation by a supervisor, PGY $2 \mathrm{~s}$ may become the supervisor of all internal medicine care overnight. Depending on the context, residents may be responsible for overseeing the ongoing care of patients in the emergency room as well as on the inpatient ward, communicating with members of the medicine and interprofessional team, all while ensuring the mentorship and learning needs of junior trainees are fulfilled. ${ }^{3}$

We know from previous research that the increase in responsibility overnight can be a difficult transition for residents to navigate. ${ }^{4-7}$ Currently, there is a paucity of literature examining transitions within residency and far less concerning transitions specific to IM programs. ${ }^{8}$ Moreover, many of the clinical activities performed overnight do not unfold in a single, observable encounter, inhibiting residents from receiving immediate, structured 
feedback on a consistent basis. ${ }^{9-11}$ The organizational and communication challenges residents encounter overnight can radically influence SMRs' response to competing oncall demands. Shifting team membership, heavy workload, indirect clinical supervision, frequent transitions in care, and team dynamics are all factors influencing the extent to which residents can meaningfully advance their learning and concurrently meet patient care expectations. ${ }^{9}, 12-16$ While existing research has sought resident perspectives on the supervisory and communication practices of the oncall SMR, ${ }^{3}$ studies have not explored this from the perspective of those healthcare professionals the SMR interact most frequently with overnight.

Thus, the purpose of this study was to elaborate in greater depth the different components of on-call SMR practice and the range of ways SMRs, effectively and less effectively, enact these. By better understanding SMRs' on-call responsibilities from multiple, relevant perspectives, we hope supervisors and training programs will be better positioned to design assessment tools that meaningfully assess resident competence and development.

\section{METHODS}

We used a constructivist grounded theory (CGT) approach to explore on-call SMR practice and what assessment of this role should consist of. ${ }^{17}$ Grounded theory was an appropriate choice because it seeks to generate a rich theoretical account of studied phenomena by exploring how a social process, such as enactment of the on-call SMR role, manifests. ${ }^{18}$ In alignment with CGT principles, we acknowledge the interpretive nature of theory generation and thus our goal was to articulate what Charmaz (2014) calls an "imaginative understanding"17 of the studied phenomenon rather than advance an explanation that emphasizes universality. To do this, we collected and analyzed data concurrently using the constant comparison method. ${ }^{17}$

\section{Setting}

This study took place in the emergency room and in-patient clinical teaching unit (CTU) of an academic health sciences center (AHSC) in Ontario, Canada. In this context, patients are typically admitted by the on-call junior trainees (senior medical students and PGY 1 residents) under the supervision of the SMR with attending physicians available for case review by telephone.

\section{Data Collection}

Data collection took place from March 2016 until February 2017. Recruitment occurred via email; faculty members, junior trainees, and nursing staff were sent an invitation to participate in on-shift note-taking over a period of 1-7 shifts and in a follow-up individual interview. These groups were identified because, excluding patients, they make up the majority of individuals that the SMRs interact with on call and they could each draw attention to different components of on-call SMR practice and therefore reveal unique perspectives on different aspects of resident competency. While RNs working in the inpatient unit were also considered, because they largely interact with the junior trainees, we did not try to enroll them. Participants $(n=24)$ included six medical students, five junior residents, five IM attending physicians, five ER physicians, and three ER nurses.

To prepare for on-shift note-taking, S.B. introduced participants to the project purpose and oriented them to the structured observation form they would complete. Participants were instructed to take detailed, anonymous notes about their experiences and observations of working with SMRs, ideally across multiple on-call periods. The number of observations each participant completed was based on workflow and scheduling considerations. The SMRs featured in participants notes were based on who was on-call during the observation periods. Participants were then interviewed by S.B. with the observation notes used as a reference tool for participant recall. Recognizing that there is wide variability in how SMRs approach overnight call, we purposely did not limit discussion to a "type" of SMR (e.g., strong versus weak). Instead, participants were asked to describe the practices of the on-call SMRs they interacted with overnight and the extent to which these practices appeared to be effective. Given that assessments are often performed based on social comparisons, ${ }^{19}$ participants were additionally asked to provide comparative reflections on SMRs they worked with previously. Observation forms and interview questions were tailored to each group as we anticipated that SMR practice would be characterized differently depending on the level of, and reason for, interaction with the SMR (see Appendices). All interviews were audio-recorded, transcribed, and de-identified.

\section{Data Analysis}

The analytic process followed a three-phase coding process. First, the research team (S.B., S.C., and M.G.) engaged in initial coding, whereby each team member independently coded the first three transcripts line-by-line to identify frequently occurring ideas and actions in the data. Together, the team consolidated initial codes and moved to the second coding phase, focused coding, where the most recurrent initial codes were assessed for their utility in advancing toward more robust theoretical categories. During this phase, certain codes were subsumed by others as unifying features were identified. This moved us into our last phase, theoretical coding, where we mapped relationships between categories, "conceptualizing our data rather than simply categorizing it". ${ }^{20}$ The team met regularly to discuss initial impressions of the data, to refine codes, and to determine which concepts were most central and compelling to the research story. Analytic memowriting occurred throughout all phases of the research; these 
critical reflections were integral to generating new insights and enhancing rigor. ${ }^{17,21}$ Data collection and analysis ended when we determined the quality of data gathered was sufficient in achieving conceptual depth. ${ }^{22}$ Investigator triangulation ${ }^{23}$ brought distinctly different perspectives to this study which proved to be a strength in ensuring the trustworthiness of the study findings. S.B. is a $\mathrm{PhD}$ trained medical education researcher with distinct experience conducting qualitative research. S.C. is a $\mathrm{PhD}$ trained education researcher working in measurement and evaluation. M.G. is a $\mathrm{PhD}$ trained medical education researcher who also works as a specialist of internal medicine. Western University Health Science Research Ethics Board provided an exemption for this phase of the research study due to the quality assurance nature of the study.

\section{RESULTS}

Our analysis revealed that SMRs with an adeptness at applying non-technical skills to complex clinical and interpersonal situations overnight were highly regarded by participants. Furthermore, what separated a perceived effective SMR from a less effective one was the added commitment some SMRs made to ensuring the tasks they performed overnight were done right. According to participants, "doing it right" required forethought, involving others in their planning, and communicating clear expectations regardless of high workplace demands. As we unpacked this notion of doing it right further, we settled on the following three components of SMR practice that, based on what participants described, enabled some SMRs to make a lasting impression. Perceived effective SMRs understood their role and responsibilities in relation to the roles of their colleagues and had a repertoire of strategies for negotiating these; worked earnestly to build relationships with other healthcare professionals; and exhibited admirable organizational skills. Perceived less effective SMRs, while potentially also having issues with clinical skills, appeared to stand out more when they could not effectively manage the realities of collaboration in a busy workplace. In what follows, the use of the terms "effective" and "less effective" is specific to the on-call role and cannot be correlated to other aspects of SMR performance.

\section{Roles, Responsibilities, and Repertoires}

Effective SMRs were described as having a broad repertoire of strategies that they used to navigate the environmental stresses or high acuity situations they encountered overnight. Emergency physicians, for example, placed high value on timely and effective communication overnight:

One of the things I've seen a couple of residents do over the years is when they're going to the floor to do something that's going to take them a while.... they'll actually come and say, I've got to go to the floor for 45 minutes, do you have anything pending because I'm not going to be able to answer my pager during that time. That's nice having a heads-up, knowing I don't have to page them every 15 minutes. (ER Physician 3)

Similarly, ER nurses recognized that sharing information was essential to supporting one another's work overnight: "The more they communicate, the more we can prompt, suggest and advocate for the patient and help the SMR out." (ER RN 1)

Supporting one another's work also required SMRs to gauge each junior team member's comfort and experience levels. Junior trainees often described the role of on-call SMR as one of a coach or mentor. Particularly for medical students, empowering them to take on meaningful roles while still being there to provide the needed expertise and back-up appeared to be a dominant feature distinguishing perceived effective SMRs:

She was probably the most unstable patient that I had seen up until then ...It was just helpful to have the SMR up there making more the important decisions. He came up right away and assessed the patient. And then, because she looked so ill, he got some stuff started, and then also called the family to tell them what was going on, and to discuss what sort of interventions they wanted and didn't want ...he still asked for my opinion on how things were going...so, he was still involving me. (Medical Student 3)

SMRs deemed less effective were described as taking a more individualistic approach to their overnight responsibilities. For ER physicians, this became visible during consults: “A weaker SMR will be obstructive, will say I don't believe this patient needs admission... and it can come down to SMRs refusing to see patients." (ER Physician 1)

ER nurses identified less effective SMRs as those who "talked over the patients' heads and their level of understanding." (ER RN 3) Similarly, junior trainees, described less effective SMRs as those who "don't even want to hear how you would do things." (Junior Resident 1)

\section{The Importance of Building Relationships}

The quality of relationships between the on-call SMR and colleagues overnight also appeared to impact an SMR's perceived effectiveness: "Most of my strength and weaknesses don't come down to knowledge base ... it comes down to communication skills, collegiality and understanding where I am when I'm talking to them on the telephone." (ER Physician 1)

ER nurses highlighted enthusiasm as another important quality high-performing SMRs appeared to possess: "She [the SMR] spends fantastic time with the patients, the families, the nursing staff. She makes herself available, making a point of giving the nurses her pager and saying, call me for anything." (ER RN 3) 
Effective SMRS were described by juniors as "approachable and collaborative.... where you don't feel like every time you ask them a question, it's like, oh, this again." (Junior Resident 2) Juniors also appreciated when the SMR would acknowledge their contributions overnight: "I've had SMRs the next day send you a quick message and be like, hey, you did a great job last night. And, that makes a big difference." (Junior Resident 1)

In many ways, it was this notion of "basic" good manners that strengthened the SMR's relationship with emergency room colleagues, co-residents, and medical students; they were often spontaneous in nature and appeared easy to do. However, because they can easily be overlooked, they were easy to neglect. Participants identified tone and particular nonverbal behaviors, "they'll just zoom in, zoom out" (ER RN 3) as negatively impacting interpersonal dynamics overnight. Strained interactions with the SMR were most often described by healthcare staff in the emergency department: "I remember one night, it was like 2:00 in the morning before I figured out who the SMR was. There were residents all over the place... but I didn't know who the SMR was." (ER RN 1)

Emergency physicians echoed the impact of negative SMR behavior overnight:

If you have a negative interaction where they push back, you hang up the phone and automatically you're critical...that kind of sours you for a little while...I find if I make a consult and we have a great conversation, it affects my practice in the sense that if I have other patients later in the night, I'm not afraid to call. (ER Physician 3)

Often such tensions were linked to individual personalities: "He [the SMR] was a bit almost overbearing...I didn't feel like he had any confidence in me or trust in my assessment. (Junior Resident 1) Other times, such actions were attributed to workplace demands. Emergency physicians, for example, recognized the role complexity of being an SMR:

We are the people that make their night worse...I mean, we try as much as we can to make it a positive interaction and to be as helpful as we can...I think it's the worst job in the hospital truthfully. (ER Physician 5)

Patient volume was also described as significantly impacting an SMR's ability to collaborate effectively. Whereas effective SMRs maintained collegial relationships when stressed, less effective SMRs did not resulting in perceived gaps in information transfer.

\section{Strong Organizational Skills}

What constituted an effective SMR overnight also came down to strong organizational skills. Effective SMRs understood that although there are numerous tasks to perform overnight, these can be prioritized. Effective SMRs were flexible, adapting to a constantly changing priority list:

It comes back to telling you what they're prioritizing. If I say to them, I have a sick patient, it's helpful when they say, okay...I have four other people with this, this, this and this. Where do you think your patient fits? (ER Physician 3)

According to junior trainees, effective SMRs "delegate very effectively" (Junior Resident 3), "document really clearly" (Medical Student 5), and have "gone to see the patient before they give the case to you." (Junior Resident 2) They also actively share, solicit, and encourage diverse perspectives so the best course of action for the patient can be determined: " $\mathrm{A}$ lot of them will actually come and speak to the nurses right off the bat and ask what their opinion is." (ER RN 3)

For IM attending physicians, evidence of an effective SMR was made visible by how little prompting post-call residents and medical students needed during morning handover: "When a medical student can present a history so completely with so much appropriate detail, I have more confidence that the senior and the clerk talked about it because I just don't expect a clerk to think about everything." (IM Attending 3) By contrast, junior trainees working with a perceived less effective SMR appeared more disorganized in their morning case presentation: "Their problem lists are incomplete ... They are talking about a lot of irrelevant stuff in their past medical history and social history which has nothing to do with the problem list that they just spoke about it." (IM Attending 1)

While a busy night could threaten to derail an SMR's ability to remain organized, the most effective had strategies for preventing this while also supporting the trainees they were working with overnight. As one medical student recalled, "I didn't, at any time, feel overwhelmed because I knew the SMR was there to help me." (Medical Student 6) By contrast, less effective SMRs "get bogged down in little details" (Junior Resident 3) and appeared to stand out more when they could not handle the repeated task switching required in the clinical environment.

\section{Visible, Invisible, and Inferred}

The SMR role was well acknowledged by participants as being multifaceted, requiring versatility, and, at times, extremely challenging to carry out. It was also noted to be a role with many invisible components. For example, it was not until one of the junior residents was preparing to take on the SMR role that they realized the level of responsibility SMRs shouldered:

I kind of assumed it was like, so the SMR gets the call, and then they tell us, and then we go do the history and physical exam. We come back, review the case, and then it's done. I think, after...I held the SMR phone, and had a med student underneath me, and then the 
night holding the SMR pager, too, I realized that they pretty much do everything. (Junior Resident 2)

Interestingly, many components of the SMR role considered most important by other participants - collaboration, strong interpersonal skills, positive demeanor-were largely invisible to the attending physicians responsible for assessing the SMRs. Consequently, they were left to infer the quality of an SMR's performance overnight based on what they observed in the morning:

A junior, when they present something...I want enough detail in their presentation to know that they've thought about things...Now what goes into that, what happens overnight I really don't know. I don't know if it's a really good presentation because the junior is very strong or because the senior resident helped them. (IM Attending 3)

It was not uncommon for attending physicians to suspect a breakdown in communication or collaboration overnight; however, being at a distance, they were unable to elaborate on the details. As a result, attending physicians appeared to give on-call SMRs the benefit of the doubt, not assuming poor judgement when there was uncertainty around circumstances:

Who am I to say that past 14 admissions in a night, the senior resident should have done this or should have done that? That's often related to a lot of other things that draw on their time, emergencies, urgencies on the floor...really busy patients that might not have even come to us or got admitted but they took up a lot of time overnight. (IM Attending 4)

As with all of the participants, attendings also recognized that consistently enacting and making visible the qualities of an effective SMR could be difficult; workload, the strength of the juniors they were on-call with, and critically ill patients all were recognized as potentially impacting an SMR's effectiveness overnight.

\section{DISCUSSION}

Our study is the first we know of to describe the experiences of those collaborating with the SMR overnight and, more specifically, help reveal the invisible practice of how SMRs approach their on-call activities. From what our participants shared, effective SMRs, even under high stress, rely on a repertoire of communication strategies to manage challenging clinical situations and interpersonal dynamics overnight. By contrast, less effective SMRs appeared to stand out more when they could not handle the demanding nature of the on-call role. This manifested itself in several, undesirable ways: questions going unanswered, SMRs appearing unapproachable, junior residents and medical students feeling ill-supported, and poor bedside manner. By understanding and contrasting the range of practices associated with after-hours duty, we can begin to generate valuable insights into what contributes to effective practice overnight so as to better inform the design of assessments that support workplace-based learning.

The findings from this study have utility from both an assessment and training perspective. First, this work suggests directions on how training programs might begin designing assessment tools that can authentically measure an SMR's performance overnight and support the provision of formative feedback. The contribution of our findings is that they illuminate components and intricacies of the on-call role that often go unrecognized, enabling training programs to reconsider how they approach the monitoring of resident growth and readiness for increased independence.

In our study's context, the attending physician has traditionally provided most of the supervision and assessment of on-call residents. It is logical to assume then that they would bear responsibility for making entrustment decisions. However, our study results lead us to question how entrustment decisions around the SMR on-call role can confidently be made when critical aspects of resident performance overnight are invisible to IM attending physicians. We need more robust assessment methods if we are to generate a comprehensive understanding of the SMR on-call role. In the USA, the Accreditation Council for Graduate Medical Education (ACGME) already endorses the use of 360-degree assessment in residency training. In the Canadian context, 360-degree assessment is encouraged but not mandated. In both contexts, however, we would argue that the SMR on-call role requires 360-degree assessments as part of the entrustment process. Consistently implementing this method of evaluation and feedback provides insight into areas for improvement about which the resident may be oblivious. ${ }^{24}$

Second, our findings provide SMRs and supervisors with a common language to converse and reflect on more and less effective practices overnight. In turn, residency programs will be better equipped to identify relevant practice activities that appropriately prepare junior residents for transition into this role. While an assortment of informal orientation opportunities to the on-call role exist already (e.g., having a PGY 1 resident carry the SMR pager), programs should consider what programmatic efforts can be taken to ensure junior trainees are sufficiently prepared for the on-call SMR role. Existing interventions include participation in an on-call simulation, ${ }^{25}$ introduction of a "buddy-system" overnight where a more experienced SMR is paired with a newly minted SMR, ${ }^{26}$ or the use of additional "float" senior residents during the first couple of weeks of a junior resident's transition to the senior role. ${ }^{27}$ 


\section{LIMITATIONS}

There are three main limitations that we wish to draw attention to. The first is the contextual nature of the data. This was a single-center study with a particular type of SMR role. As a result, others wishing to explore the transferability of our findings will need to consider both the similarities and differences between their context and the one presented here. The second limitation relates to the overlapping nature of competencies in competent practice. For example, less effective communication and organizational enactments may not only be a problem from those perspectives but also a reflection of gaps in clinical skills; teasing out the underlying cause of less effective performance was however not the purpose of this study. Finally, while we strove to explore SMR practice from the multiple perspectives of those who interacted with them, inpatient nurses and patients and their families were not included in this study. We suggest future studies consider including their perspectives as there may be facets of the role experienced from these perspectives that differ from what is already known about effective communication and care practices during daytime hours.

\section{CONCLUSION}

Our study is timely because it invites training programs to consider what residents, particularly those in more complex roles such as the on-call SMR, need to be assessed on to support entrustment decisions and learner development. In our study's context, we found resounding attention to the importance of strong collaboration and organizational skills overnight. Moreover, we were able to describe specific effective and less effective practices that impacted both interprofessional relationships and patient care overnight. Such specificity can be helpful in the development of both formative and summative assessment tools. Finally, our identification of the invisibility of these practices to their supervising attendings once again draws attention to the need to include multiple perspectives when assessing roles of this nature.

Acknowledgments: We would like to thank the participants for their contributions to this study.

Corresponding Author: Sarah Burm, PhD; Continuing Professional Development/Division of Medical Education, Faculty of Medicine, Dalhousie University, Room 2L-23 Sir Charles Tupper Medical Building, 5850 College Street, Halifax, Nova Scotia, Canada (e-mail: Sarah.Burm@dal.ca).

Funding This study was funded by the Royal College of Physicians and Surgeons of Canada.

\section{Compliance with Ethical Standards:}

Western University Health Science Research Ethics Board provided an exemption for this phase of the research study due to the quality assurance nature of the study.
Conflict of Interest: The authors declare that they do not have a conflict of interest.

\section{REFERENCES}

1. Holmboe ES, Sherbino J, Long DM Swing SR, Frank JR. The role of assessment in competency-based medical education. Med Teach. 2010; 32(8): 676-682.

2. ten Cate O, Scheele F. (2007). Competency-based postgraduate training: Can we bridge the gap between theory and clinical practice? Acad Med.; 82(6): 542-547.

3. Huda N, Faden L, Goldszmidt M. Entrustment of the on-call senior medical resident role: Implications for patient safety and collective care. BMC Med Educ. 2017; 17(1): 121-130.

4. Walzak A. Butler D, Bates J, Farrell L, Law SFB, Pratt DD. Working in the dead of night: Exploring the transition to after-hours duty. Med Educ. 2019; 53(3): 296-305.

5. Yardley S, Westermen M, Bartlett M, Walton JM, Smith J, Peile E. The do's, don'ts, and don't knows of supporting transition to more independent practice. Perspect Med Educ. 2018; 7: 8-22.

6. Wipt JE, Pinksy LE, Burke W. Turning interns into senior medical residents: Prepearing residents for their teaching and leadership roles. Acad Med. 1995; 70(7): 591-596.

7. Bernabeo EC, Holtman MC, Ginsburg S, Rosenbaum JR, Holmboe, ES. Lost in transition: The experience and impact of frequent changes in the inpatient learning environment. Acad Med. 2011; 86(5): 591-598.

8. Khalife R, Gonsalves C, Code C, Halman S. Transitioning towards senior medical resident: Identification of the required competencies using consensus methodology. Can Med Educ J. 2018; 9(3): e64-e75.

9. Hatala R, Ginsburg S, Hauer KE, Gingrich A. Entrustment ratings in internal medicine: Capturing meaningful supervision decisions or just another rating? J Gen Intern Med. 2019; 34(5): 740-743.

10. Sebok-Syer S, Chahine S, Watling CW, Goldszmidt M, Cristancho S, Lingard L. Considering the interdepending of clinical performance: Implications for assessment and entrustment. Med Educ. 2018; 52(9): 970-980.

11. Warm EJ, Mathis BR, Held JD et al. Enturstment and mapping of observable practice activities for resident assessment. J Gen Intern Med. 2014; 29(8): 1177-1182.

12. Gingrich A, Daniels V, Farrell L, Olsen SR, Kennedy T, Hatala R. Beyond hands-on and hands-off: Supervisory approaches and entrustment on the inpatient ward. Med Educ. 2018; 52(10): 1028-1040.

13. Kennedy TJT, Lingard L, Baker RG, Kitchen L, Regehr G. Clinical oversight: Conceptualizing the relationship between supervision and safety. J Gen Intern Med. 2007; 22(8): 1080-1085.

14. Burm S, Faden $\mathbf{L}$, DeLuca S, Hibbert $\mathbf{K}$, Huda N, Goldzmidt. Using a sociomaterial approach to generate new insights into the nature of interprofessional collaboration: Findings from an inpatient medicine teaching unit. J Interprof Care. 2019; 33(2): 153-162.

15. Goldszmidt M, Dornan T, Lingard L. Progressive collaborative refinement on teams: Implications for communication practices. Med Educ. 2014; 48(3): 301-314.

16. Farnan JM, Johnson JK, Meltzer DO, Humphrey HJ, Arora VM. Oncall supervision and resident autonomy: From micromanager to absentee attending. AM J Med. 2009; 122(8): 784-788.

17. Charmaz K. Constructing Grounded Theory. London: SAGE Publications; 2014.

18. Charmaz K. The power of constructivist grounded theory for critical inquiry. Gual Inq. 2017; 23(1): 34-45.

19. Yeates P, O'Neill P, Mann K, Eva K. "You're certainly reletively competent": Assessor bias due to recent experiences. Med Educ. 2013; 47(9): 10-22.

20. Watling $\mathbf{C J}$, Lingard L. Grounded theory in medical education research: AMEE guide no. 70 Med Teach. 2012; 34(10): 850-861

21. Bradbury-Jones C. Enhancing rigour in qualitative health research: Exploring subjectivity through Peshkin's I's. J Adv Nurs. 2007; 59(3): 290-298.

22. Nelson J. Using conceptual depth criteria: Addressing the challenge of reaching saturation in qualitative research. Qual Res. 2017; 17(5): 554570.

23. Mitchell, ES. Multiple triangulation: A methodology for nursing science. Adv Nurs Sci. 1986; 8(3): 18-26. 
24. Rodgers KD, Manifold C. 360 degree feedback: Possibilities for assessment of ACGME core competencies for emergency medicine residents. Acad Emerg Med. 2002; 9(11): 1300-1304.

25. Kalet A, Zabar S, Szyld D, et al. A simulated "night on call" to assess and address the readiness-for-internship of transitioning medical students. Adv Simul. 2017; 2(3): 1-9
26. Maurice A, Hann A. Training in general surgery ward call: A residentstudent buddy system. BMJ Open Qual. 2015; 4(1): 1-3.

27. Petrilli CM, Del Valle J, Chopra V. Why July matters. Acad Med. 2016; 91(7): 910-912.

Publisher's Note: Springer Nature remains neutral with regard to jurisdictional claims in published maps and institutional affiliations. 